\title{
Open heavy-flavour production with the ALICE experiment at the LHC
}

\section{Diego STOCCO* for the ALICE Collaboration}

( Laboratoire de Physique Subatomique et des Technologies Associées )

E-mail: diego.stoccodcern.ch

\begin{abstract}
The ALICE experiment measured heavy-flavour production in a wide rapidity range in heavy-ion and proton-proton collisions at the LHC. The transverse momentum distributions of D mesons, and of heavy flavours in the semi-electronic and semi-muonic decay channels in pp collisions at 2.76 and $7 \mathrm{TeV}$ will be presented and discussed. The results are compared with perturbative quantum chromodynamics calculations: the predictions agree with data within the theoretical and experimental uncertainties.

These measurements have been used as a reference to study the effects of the high energy density matter formed in heavy-ion collisions. The resulting nuclear modification factors will also be briefly discussed.
\end{abstract}

XXI International Workshop on Deep-Inelastic Scattering and Related Subjects 22-26 April, 2013

Marseilles, France

\footnotetext{
* Speaker.
} 


\section{Introduction}

The ALICE experiment [1] is designed to study the properties of the high energy-density medium formed in ultra-relativistic heavy-ion collisions. Heavy-flavour hadrons (containing charm or beauty) are effective probes of the mechanisms of heavy-quark propagation and energy loss in the hot and dense QCD matter. Their measurement in pp collisions has a twofold interest: on the one hand it provides a reference for the study of the effects observed in heavy-ion collisions; on the other hand, it allows for a precise test of perturbative quantum chromodynamics (QCD) calculations in the high energy domain.

The ALICE experiment measures D mesons through hadronic decay channels at mid-rapidity and inclusive heavy flavours in the semi-muonic and semi-electronic decay channels at forward and central rapidity, respectively. In the latter case, the contribution of electrons from beauty decays can also be separated. The measurements are carried out through the use of several detectors allowing for tracking, triggering, vertexing and particle identification.

At mid-rapidity $(|\eta|<0.9)$, the tracks are reconstructed with the Time Projection Chamber (TPC) and the Inner Tracking System (ITS). The detectors are immersed in a 0.5 T magnetic field, allowing for track measurement down to low transverse momenta. The ITS consists of six layers of silicon detectors, allowing for a resolution on the track impact parameter (distance between the track and the primary vertex) of better than $75 \mu \mathrm{m}$ for $p_{T}>1 \mathrm{GeV} / c$ [2]. The particle identification is performed by combining the information of the specific energy loss of particles in the TPC gas and the one of the Time Of Flight detector. In addition, the Transition Radiation Detector (TRD) and the Electromagnetic Calorimeter (EMCal) are used for electron identification. Muons are reconstructed in the Forward Muon Spectrometer, in the pseudo-rapidity range $-4<\eta<-2.5$. As seen from the interaction point, the Spectrometer consists of a front absorber, five stations of tracking chambers, an iron wall and two stations of trigger chambers. Tracks are bent by a dipole magnet providing an integrated magnetic field of $3 \mathrm{~T} \times \mathrm{m}$. Muon identification is performed by matching a track in the tracker with a tracklet in the trigger chambers, placed downstream an iron wall of about 7.2 interaction lengths thickness [1].

The data sample for D meson and heavy-flavour electron analyses consists of Minimum-Bias (MB) events, which are defined using the information of the Silicon Pixel Detector (SPD) and the VZERO detector. The former constitutes the two innermost layers of the ITS, while the latter consists of two arrays of scintillator tiles with full azimuthal coverage in the pseudo-rapidity regions $2.8<\eta<5.1$ and $-3.7<\eta<-1.7$. MB collisions were triggered by requiring at least one hit in the SPD or in either of the VZERO counters in coincidence with the arrival of proton bunches in both sides. The muon trigger requires, in addition to the MB condition, the presence of a muon with a transverse momentum higher then $0.5 \mathrm{GeV} / c$ in the muon trigger chambers.

\section{D mesons in the hadronic decay channel}

Charm production was measured in pp collisions at $\sqrt{s}=2.76$ and $7 \mathrm{TeV}$ through the exclusive reconstruction of three decay modes for the D mesons: $D^{0} \rightarrow K^{-} \pi^{+}, D^{+} \rightarrow K^{-} \pi^{+} \pi^{+}$and $D^{*+} \rightarrow D^{0} \pi^{+} \rightarrow K^{-} \pi^{+} \pi^{+}$and their charge conjugate. In addition, the charm-strange meson was measured in the decay channel $D_{s}^{+} \rightarrow \phi \pi^{+} \rightarrow K^{+} K^{-} \pi^{+}$and its charge conjugate in pp collisions 
at $\sqrt{s}=7 \mathrm{TeV}$. The analysis strategy is based on the reconstruction and selection of secondary vertex topologies that have significant separation from the primary vertex. The identification of kaons, performed with the TPC and TOF detectors, allows to further reduce the background at low momenta. Finally, the signal is extracted through a fit of the invariant mass distribution. The resulting $\mathrm{D}$ meson yield consists of a prompt contribution and a contribution from the feed-down of B mesons. The latter was estimated via perturbative QCD calculations in the framework of the Fixed-Order Next-To-Leading Log (FONLL) [3] and subtracted.

The transverse momentum distribution of prompt $\mathrm{D}$ mesons was compared to perturbative QCD calculations, which well describe data in pp collisions both at $\sqrt{s}=2.76 \mathrm{TeV}$ [4] and $7 \mathrm{TeV}$ [2, 5]. For illustration, the results for the $D^{0}$ and $D_{s}^{+}$mesons are shown in Figure 1.
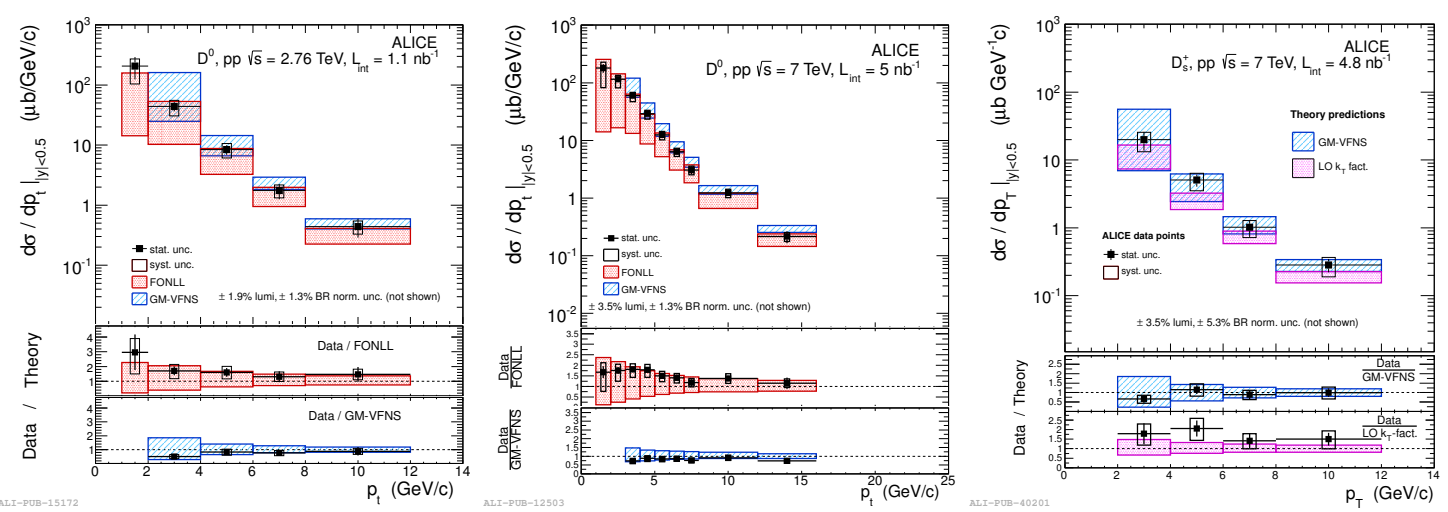

Figure 1: Transverse momentum distribution of $D^{0}$ mesons in pp collisions at $\sqrt{s}=2.76 \mathrm{TeV}$ (left panel) [4] and $7 \mathrm{TeV}$ (middle panel) [2], and of $D_{s}^{+}$in pp collisions at $\sqrt{s}=7 \mathrm{TeV}$ (right panel) [5]. The results are compared to perturbative QCD calculations (see $[2,4,5]$ and references therein). The ratio of data over theory is shown in the bottom panel of each figure.

\section{Heavy-flavour decay electrons}

The identification of electrons at mid-rapidity is performed with high purity using different detectors [6]. The information of the specific energy loss in the TPC is used in all approaches, in combination with the particle time of flight in order to resolve the ambiguities at low momentum, where the electron, kaon, proton and deuteron lines cross. At high transverse momenta, the hadron contamination is further suppressed by using the TRD or the Electromagnetic Calorimeter information. The inclusive transverse momentum distribution of electrons consists of many sources other than heavy-flavour decay. The most important are the Dalitz decay of light neutral mesons, di-electron decays of vector mesons and quarkonia, photon conversion in the beam-pipe and detector materials, and direct radiation. Such contributions have been statistically subtracted from the inclusive spectrum through a Monte Carlo cocktail based on the measured distributions of $\pi^{0}$, with the resonance decay contributions obtained through $m_{T}$ scaling and the direct $\gamma$ contribution derived from Next-to-Leading Order (NLO) perturbative QCD calculations [6].

The results for pp collisions at $\sqrt{s}=7 \mathrm{TeV}(2.76 \mathrm{TeV})$ are shown in the left (middle) panel of Figure 2. For the $7 \mathrm{TeV}$ case, the ATLAS results [7] are shown together with the ALICE ones, 
thus illustrating the complementarity of the two experiments in the explored kinematic range. The results are compared with the FONLL calculations [3], which well describe data at both energies.
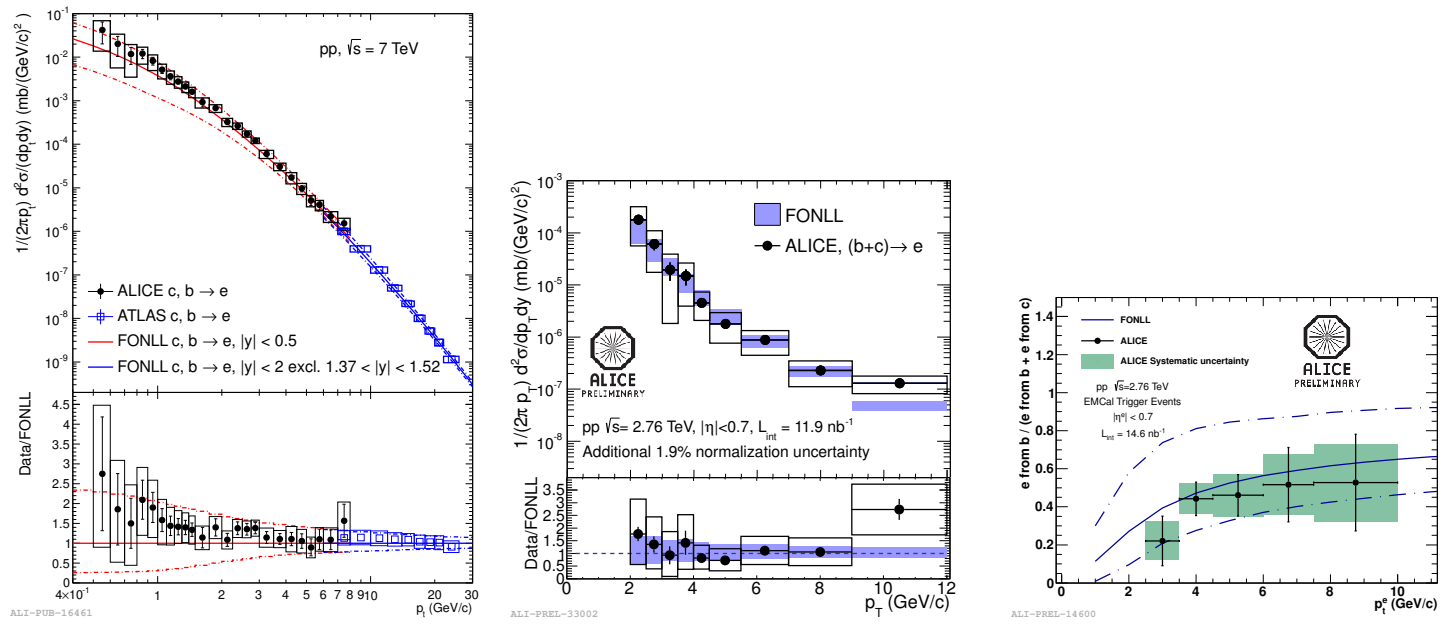

Figure 2: Transverse momentum distribution of electrons from heavy-flavour decays in pp collisions at $\sqrt{s}=7 \mathrm{TeV}$ (left panel) [6] and 2.76 TeV (middle panel). The results are compared to FONLL predictions [3]. The ratio of data over theory is shown in the bottom panel of each figure: a good agreement is found. In the left panel, the ATLAS results [7] are also shown (blue points). Right panel: fraction of electrons from beauty decays in the inclusive heavy-flavour electron distribution measured in pp collisions at $\sqrt{s}=2.76 \mathrm{TeV}$.

The described measurements treat the charm and beauty production inclusively. It is however possible to further separate the two contributions, using two different methods. The first method exploits the larger lifetime of B mesons compared to D meson, resulting in a larger distance of the decay vertex from the primary vertex. A cut on the impact parameter of the decay products is applied to enrich the beauty contribution. The remaining contamination from charm is estimated using the measured D meson distributions (see previous section) and subtracted [8]. The second method exploits the azimuthal angular correlation of electrons and charged hadrons on the near side. Due to the larger mass, the correlation distribution of the decay products of beauty is wider than the one of charm. A fit with Monte Carlo templates can thus be used to separate the two contributions. The result of this method for pp collisions at $2.76 \mathrm{TeV}$ is shown in the right panel of Figure 2. Similar results were obtained for pp collisions at $7 \mathrm{TeV}$ [8]: the comparison with FONLL calculations shows a good agreement at both energies.

\section{Heavy-flavour decay muons at forward rapidity}

Muons are reconstructed in the Muon Spectrometer and identified by requiring a matching with a tracklet in the trigger chambers, placed behind an iron wall. The main source of background consists of muons coming from the decay in flight of light hadrons. This contribution dominates the inclusive yields at low transverse momenta, but its relative importance decreases with $p_{\mathrm{T}}$. Hence, a cut at $p_{T}>2 \mathrm{GeV} / c$ was applied. The remaining contamination was subtracted through the use of Monte Carlo simulations. The transverse momentum distributions of muons from heavy-flavour decays in pp collisions at $\sqrt{s}=7 \mathrm{TeV}(2.76 \mathrm{TeV})$ is shown in the left (right) panel of Figure 3. The comparison with FONLL [3] shows a good agreement with data at both energies. 

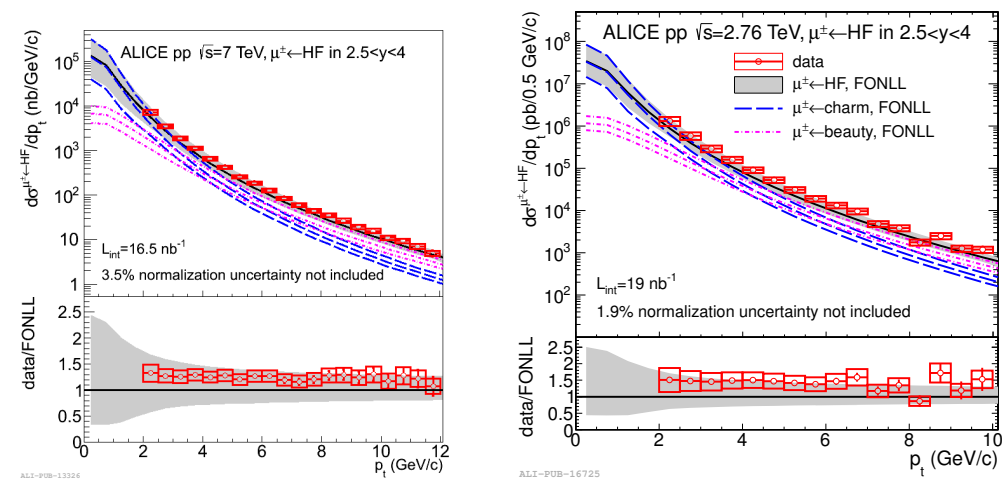

Figure 3: Transverse momentum distribution of muons from heavy-flavour decays in pp collisions at $\sqrt{s}=7 \mathrm{TeV}$ [9] and $2.76 \mathrm{TeV}$ [10]. The results are compared to FONLL predictions (grey band) [3]. The ratio of data over theory is shown in the bottom panel of each figure: a good agreement is found.

\section{Heavy flavours in heavy-ion collisions}

Heavy-flavour production was measured in $\mathrm{Pb}-\mathrm{Pb}$ collisions at a centre of mass energy of $2.76 \mathrm{TeV}$ per nucleon pair. The analysis strategy is analogous to the one described in sections 2 , 3 and 4 (see also $[10,11]$ for further details). The resulting transverse momentum distributions in heavy-ion collisions are then rescaled by the average number of binary nucleon-nucleon collisions and divided by the same quantity measured in proton-proton collisions. This observable is called the nuclear modification factor $\left(R_{A A}\right)$ and is expected to be equal to 1 if no nuclear modification is present.

The results for heavy-flavour measurements in the different decay channels are summarised in Figure 4: a large reduction of the yields of D mesons (left panel) and heavy-flavour electrons and muons (right panel) is observed in $\mathrm{Pb}-\mathrm{Pb}$ collisions with respect to $\mathrm{pp}$ ones in the high- $p_{\mathrm{T}}$ region. It is worth noting that energy loss is not the only mechanism that could lead to a modification of the $R_{A A}$. Initial-state effects, such as the variation of the parton distribution functions of nucleons inside the nuclei compared to the ones of nucleons in vacuum (shadowing), could result in a $R_{A A}$ value different than 1 . However, these effects are expected to be small in this high- $p_{T}$ region and cannot explain the observed suppression, which is therefore a consequence of the interaction with the medium.

\section{Conclusion}

The ALICE experiment measured heavy-flavour production in the hadronic and semi-leptonic decay channels in pp collisions at $\sqrt{s}=2.76$ and $7 \mathrm{TeV}$ and in $\mathrm{Pb}-\mathrm{Pb}$ collisions at a centre of mass energy of $2.76 \mathrm{TeV}$ per nucleon pair. The proton-proton results are well described by perturbative QCD calculations.

$\mathrm{In} \mathrm{Pb}-\mathrm{Pb}$ collisions, the nuclear modification factors show a strong reduction at high transverse momenta, where initial-state effects are expected to be small. A better insight of such effects at low$p_{\mathrm{T}}$ will be soon available from the analysis of the proton-ion collisions performed at the beginning of 2013. 

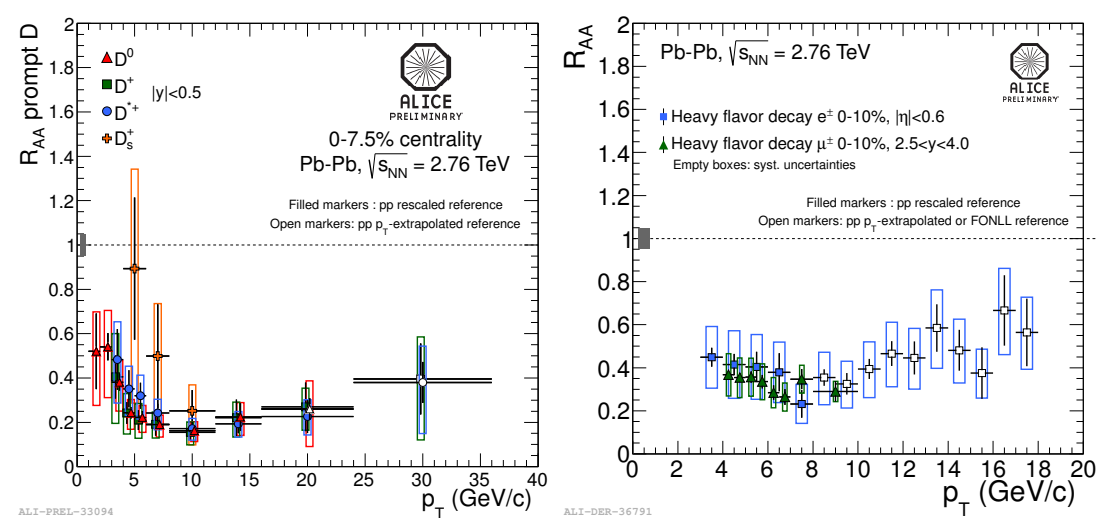

Figure 4: Nuclear modification factors of D mesons (left panel) and heavy-flavour decay leptons (right panel) in the most central $\mathrm{Pb}-\mathrm{Pb}$ collisions at $\sqrt{s}=2.76 \mathrm{TeV}$.

\section{References}

[1] K. Aamodt et al. [ALICE Collaboration], JINST 3 (2008) S08002.

[2] B. Abelev et al. [ALICE Collaboration], JHEP 1201 (2012) 128

[3] M. Cacciari et al., JHEP 1210 (2012) 137

[4] B. Abelev et al. [ALICE Collaboration], JHEP 1207 (2012) 191

[5] B. Abelev et al. [ALICE Collaboration], Phys. Lett. B 718 (2012) 279

[6] B. Abelev et al. [ALICE Collaboration], Phys. Rev. D 86 (2012) 112007

[7] G. Aad et al. [ATLAS Collaboration], Phys. Lett. B 707 (2012) 438

[8] B. Abelev et al. [ALICE Collaboration], Phys. Lett. B 721 (2013) 13

[9] B. Abelev et al. [ALICE Collaboration], Phys. Lett. B 708 (2012) 265

[10] B. Abelev et al. [ALICE Collaboration], Phys. Rev. Lett. 109 (2012) 112301

[11] B. Abelev et al. [ALICE Collaboration], JHEP 1209 (2012) 112 